\title{
PERIODIC ORBITS OF THE PLANAR ANISOTROPIC MANEV PROBLEM AND OF THE PERTURBED HYDROGEN ATOM PROBLEM
}

\author{
JAUME LLIBRE ${ }^{1}$ AND PENGFEI YUAN ${ }^{2}$
}

\begin{abstract}
In this paper we use the averaging theory for studying the periodic solutions of the planar anisotropic Manev problem and of two perturbations of the hydrogen atom problem. When a convenient parameter is sufficiently small we prove that for every value $e \in(0,1)$ a unique elliptic periodic solution with eccentricity $e$ of the Kepler problem can be continued to the mentioned three problems.
\end{abstract}

\section{INTRODUCTION AND STATEMENT OF THE RESULTS}

The Manev problem is a two-body problem with a potential of the following form

$$
V(r)=\frac{a}{r}+\frac{b}{r^{2}},
$$

where $r$ is the distance between the two particles and $a, b$ are arbitrary constants.

For $a, b>0$ the problem was first considered by Newton. Manev systems were recently reconsidered in a series of studies having as their starting point Diacu's research [12], see also [2],[6],[17],[20]-[23], [25][29],[35]-[38].

Abouelmagd et al. [1] study the periodic solutions for the planar anisotropic Kepler problem using averaging theory. Motivated by this work, we study the anisotropic Manev problem with $a$ and $b$ arbitrary using the averaging theory.

The equations of motion are

$$
q_{i}^{\prime}=\frac{\partial H}{\partial p_{i}}, \quad p_{i}^{\prime}=-\frac{\partial H}{\partial q_{i}}, \quad \text { for } i=1,2,
$$

Key words and phrases. periodic solutions, averaging theory, anisotropic Manev problem, hydrogen atom problem. 
(2) $H\left(q_{1}, q_{2}, p_{1}, p_{2}\right)=\frac{1}{2}\left(p_{1}^{2}+p_{2}^{2}\right)-\frac{1}{\sqrt{(1+\varepsilon) q_{1}^{2}+q_{2}^{2}}}-\frac{\varepsilon}{(1+\varepsilon) q_{1}^{2}+q_{2}^{2}}$.

Here $|\varepsilon|>0$ denotes a small parameter and the prime denotes the derivative with respect to the time $t$. The Hamiltonian system (1) is defined for all $\left(q_{1}, q_{2}, p_{1}, p_{2}\right) \in \mathbb{R}^{4}$ except in the plane $q_{1}=q_{2}=0$.

In this paper we study analytically the periodic solutions of the planar anisotropic Manev problem using the averaging theory, and we get the following result.

Theorem 1. For $\varepsilon \neq 0$ sufficiently small a unique elliptic periodic solution of the Kepler problem for each value of the eccentricity can be continued to the energy level $h(e)$ of the planar anisotropic Manev problem (1), where

$$
h(e)=-\frac{\left(1-e^{2}\right)\left(1-\sqrt{1-e^{2}}\right)^{2}}{4 e^{4}} .
$$

The proof of Theorem 1 is given in Section 2 .

The hydrogen atom interaction with a circular polarized microwave field is a quiet well known problem in classical mechanics. There are extensive literatures on this problem, for more details, see $[3,4],[7,8$, 9], [13]-[16], [18, 19], [30]-[33] , [39].

In [31] the authors studied the hydrogen atom problem in a rotation frame with the following Hamiltonian

$$
H=\frac{1}{2}\left(p_{x}^{2}+p_{y}^{2}\right)-x p_{y}+y p_{x}-\frac{1}{\sqrt{x^{2}+y^{2}}}+\varepsilon x .
$$

We consider the same potential but in fixed coordinates, and instead of the perturbation $\varepsilon x$ with two more general perturbations $\varepsilon\left(a x+b x^{2}\right)$ and $\varepsilon\left(a x+b y^{2}\right)$.

For the perturbation $\varepsilon\left(a x+b x^{2}\right)$ the corresponding Hamiltonian system is

$$
\begin{aligned}
x^{\prime} & =p_{x}, \\
y^{\prime} & =p_{y}, \\
p_{x}^{\prime} & =-\frac{x}{r^{3}}-\varepsilon(a+2 b x), \\
p_{y}^{\prime} & =-\frac{y}{r^{3}},
\end{aligned}
$$

with Hamiltonian

$$
H=\frac{1}{2}\left(p_{x}^{2}+p_{y}^{2}\right)-\frac{1}{\sqrt{x^{2}+y^{2}}}+\varepsilon\left(a x+b x^{2}\right) .
$$


For the perturbation $\varepsilon\left(a x+b y^{2}\right)$ the following Hamiltonian system becomes

$$
\begin{aligned}
x^{\prime} & =p_{x}, \\
y^{\prime} & =p_{y}, \\
p_{x}^{\prime} & =-\frac{x}{r^{3}}-\varepsilon a, \\
p_{y}^{\prime} & =-\frac{y}{r^{3}}-\varepsilon 2 b y,
\end{aligned}
$$

with Hamiltonian

$$
H=\frac{1}{2}\left(p_{x}^{2}+p_{y}^{2}\right)-\frac{1}{\sqrt{x^{2}+y^{2}}}+\varepsilon\left(a x+b y^{2}\right) .
$$

We have the following two results.

Theorem 2. For $h<0,0<\left|\frac{3 a h}{4 b}\right|<1,4 b+3 a h \neq 0$ and $\varepsilon \neq 0$ sufficiently small a unique elliptic periodic solution with eccentricity $e=|3 a h /(4 b)|$ of the Kepler problem can be continued to the energy level $H=h$ of the hydrogen atom problem (3).

Theorem 3. For $h<0,0<\left|\frac{3 a h}{b}\right|<1, b-3 a h \neq 0$ and $\varepsilon \neq 0$ sufficiently small a unique elliptic periodic solution with eccentricity $e=|3 a h / b|$ of the Kepler problem can be continued to the energy level $H=h$ of the hydrogen atom problem (4).

Theorems 2 and 3 are proved in sections 3 and 4 , respectively.

\section{Proof of Theorem 1}

We change the equations of motion (1) to the McGehee coordinates $(r, \theta, v, u)$ as follows

$$
\begin{aligned}
& \left(q_{1}, q_{2}\right)=r(\cos \theta, \sin \theta) \\
& r^{-1 / 2} v=\left(p_{1}, p_{2}\right) \cdot(\cos \theta, \sin \theta) \\
& r^{-1 / 2} u=\left(p_{1}, p_{2}\right) \cdot(-\sin \theta, \cos \theta)
\end{aligned}
$$


for more details on these coordinates see $[10,11,24]$. So in the McGehee coordinates the equations of motion (1) write

$$
\begin{aligned}
& r^{\prime}=r^{-1 / 2} v, \\
& \theta^{\prime}=r^{-3 / 2} u, \\
& v^{\prime}=r^{-3 / 2}\left(u^{2}+\frac{1}{2} v^{2}+V_{1}(\theta)\right)+2 r^{-5 / 2} V_{2}(\theta), \\
& u^{\prime}=r^{-3 / 2}\left(-\frac{1}{2} u v-\frac{d V_{1}(\theta)}{d \theta}\right)-r^{-5 / 2} \frac{d V_{2}(\theta)}{d \theta},
\end{aligned}
$$

where

$$
V_{1}(\theta)=-\frac{1}{\sqrt{1+\varepsilon \cos ^{2} \theta}}, \quad V_{2}(\theta)=-\frac{\varepsilon}{1+\varepsilon \cos ^{2} \theta},
$$

and the energy level $H=h$ becomes

$$
\frac{r}{2}\left(u^{2}+v^{2}\right)+r V_{1}(\theta)+V_{2}(\theta)=r^{2} h .
$$

Note that the equations of motion (5) are defined for all $(r, \theta, v, u) \in$ $(0,+\infty) \times \mathbb{S}^{1} \times \mathbb{R}^{2}$ with a collision singularity at $r=0$. We can remove this singularity with the change $t \rightarrow \tau$ of the independent variable given by $d t / d \tau=r^{5 / 2}$. The equations of motion (5) in the new time $\tau$ become

$$
\begin{aligned}
\dot{r} & =r^{2} v, \\
\dot{\theta} & =r u \\
\dot{v} & =r\left(u^{2}+\frac{1}{2} v^{2}+V_{1}(\theta)\right)+2 V_{2}(\theta), \\
\dot{u} & =r\left(-\frac{1}{2} u v-\frac{d V_{1}(\theta)}{d \theta}\right)-\frac{d V_{2}(\theta)}{d \theta},
\end{aligned}
$$

here the dot denotes derivative with respect to $\tau$. Now the equations of motion (7) are defined for all $(r, \theta, v, u) \in[0,+\infty) \times \mathbb{S}^{1} \times \mathbb{R}^{2}$.

Since if $\varepsilon=0$ the Hamiltonian (2) of the anisotropic Manev problem becomes the Hamiltonian of the Kepler problem, we want to detect the periodic solutions of the Kepler problem which can be continued to a fix negative energy level $H=h<0$ of the anisotropic Manev problem.

Using (6) from the energy level $H=h<0$ we obtain

$$
r=r(\theta, v, u, h)=f_{0}+\varepsilon f_{1}+O\left(\varepsilon^{2}\right),
$$

where

$$
\begin{aligned}
& f_{0}=\frac{u^{2}+v^{2}-2}{2 h}, \\
& f_{1}=\frac{\left(u^{2}+v^{2}-2\right)(1+\cos 2 \theta)-8 h}{4 h\left(u^{2}+v^{2}-2\right)} .
\end{aligned}
$$


Now taking as the new independent variable the variable $\theta$, the equations of motion (7) restricted to the energy level $H=h<0$ become

$$
\begin{aligned}
& \frac{d v}{d \theta}=\frac{2\left(u^{2}+v^{2}-2\right)}{2 u}+\varepsilon \frac{\left(u^{2}+v^{2}-2\right) \cos ^{2} \theta-8 h}{2 u\left(u^{2}+v^{2}-2\right)}+O\left(\varepsilon^{2}\right), \\
& \frac{d u}{d \theta}=-\frac{v}{2}+\varepsilon \frac{\cos \theta \sin \theta}{u}+O\left(\varepsilon^{2}\right) .
\end{aligned}
$$

It is clear that computing periodic solutions of system (8), we are obtaining periodic solutions of system (1) in the energy level $H=h<0$.

The unperturbed system when $\varepsilon=0$ is

$$
\frac{d v}{d \theta}=\frac{2 u^{2}+v^{2}-2}{2 u}, \quad \frac{d u}{d \theta}=-\frac{v}{2},
$$

which has the general solution

$$
\left(v\left(\theta ; e, \theta_{0}\right), u\left(\theta ; e, \theta_{0}\right)\right)=\left(\frac{e \sin \left(\theta-\theta_{0}\right)}{\sqrt{1+e \cos \left(\theta-\theta_{0}\right)}}, \sqrt{1+e \cos \left(\theta-\theta_{0}\right)}\right) .
$$

This is the solution of the planar Kepler problem with eccentricity $e$ and argument of the pericenter $\theta_{0}$, i.e. $\theta_{0}$ is the angle which provides the direction of the pericenter. Of course, for $e=0$ this solution is circular, and for $e \in(0,1)$ it is elliptic, for more details see [34].

We are interested in knowing what are the periodic solutions of the Kepler problem which can be continued to periodic solutions of the anisotropic Manev problem, i.e. what solutions (10) with eccentricity $e \in[0,1)$ can be extended.

In order to apply the averaging theory to system (8) we identify our variables with those of averaging theory given in [5], and we obtain

$$
\begin{aligned}
& z=\left(\begin{array}{c}
e \\
\theta_{0}
\end{array}\right), \quad\left(\begin{array}{c}
t \\
T
\end{array}\right)=\left(\begin{array}{c}
\theta \\
2 \pi
\end{array}\right), \\
& F_{0}=\left(\begin{array}{c}
F_{01} \\
F_{02}
\end{array}\right), \quad F_{1}=\left(\begin{array}{c}
F_{11} \\
F_{12}
\end{array}\right), \\
& x(t ; z, 0)=\left(\begin{array}{l}
v\left(\theta ; e, \theta_{0}\right) \\
u\left(\theta ; e, \theta_{0}\right)
\end{array}\right),
\end{aligned}
$$

where

$$
\begin{array}{ll}
F_{01}=\frac{2 u^{2}+v^{2}-2}{2 u}, & F_{11}=\frac{-16 h+\left(-4+2 u^{2}+2 v^{2}\right) \cos ^{2} \theta}{4 u\left(-2+u^{2}+v^{2}\right)}, \\
F_{02}=-\frac{v}{2}, & F_{12}=\frac{\cos \theta \sin \theta}{u} .
\end{array}
$$


The first variational equation of the unperturbed system (9) along the periodic solution (10) with $e \in[0,1)$ is

$$
\left(\begin{array}{ll}
y_{1}^{\prime} & y_{2}^{\prime} \\
y_{3}^{\prime} & y_{4}^{\prime}
\end{array}\right)=J\left(\begin{array}{ll}
y_{1} & y_{2} \\
y_{3} & y_{4}
\end{array}\right)
$$

where

$$
J=D_{x} F_{0}(t, x(t ; z, 0))=\left.\left(\begin{array}{ll}
\frac{\partial F_{01}}{\partial v} & \frac{\partial F_{01}}{\partial u} \\
\frac{\partial F_{02}}{\partial u} & \frac{\partial F_{02}}{\partial v}
\end{array}\right)\right|_{v=v\left(\theta ; e, \theta_{0}\right), u=u\left(\theta ; e, \theta_{0}\right)},
$$

and consequently

$$
J=\left(\begin{array}{cc}
\frac{e \sin \left(\theta-\theta_{0}\right)}{1+e \cos \left(\theta-\theta_{0}\right)} & \frac{3}{2}+\frac{1-e^{2}}{2\left(1+e \cos \left(\theta-\theta_{0}\right)\right)^{2}} \\
-\frac{1}{2} & 0
\end{array}\right) .
$$

The Fundamental matrix $M_{z}(\theta)$ of system (11) such that $M_{z}(0)$ is the identity matrix is

$$
M_{z}(\theta)=\left(\begin{array}{ll}
y_{1}\left(\theta ; e, \theta_{0}\right) & y_{2}\left(\theta ; e, \theta_{0}\right) \\
y_{3}\left(\theta ; e, \theta_{0}\right) & y_{4}\left(\theta ; e, \theta_{0}\right)
\end{array}\right),
$$

where

$$
\begin{aligned}
& y_{1}\left(\theta ; e, \theta_{0}\right)=\frac{\sqrt{1+e \cos \theta_{0}}\left[4 \cos \theta+e\left[\cos \left(2 \theta-\theta_{0}\right)+3 \cos \theta_{0}\right]\right]}{4\left[1+e \cos \left(\theta-\theta_{0}\right)\right]^{3 / 2}}, \\
& y_{2}\left(\theta ; e, \theta_{0}\right)=\frac{q(\theta)}{4\left[1+e \cos \left(\theta-\theta_{0}\right)\right]^{3 / 2} \sqrt{1+e \cos \theta_{0}}}, \\
& y_{3}\left(\theta ; e, \theta_{0}\right)=-\frac{\sin \theta \sqrt{1+e \cos \theta_{0}}}{2 \sqrt{1+e \cos \left(\theta-\theta_{0}\right)}}, \\
& y_{4}\left(\theta ; e, \theta_{0}\right)=\frac{e \sin \theta \sin \theta_{0}+2 \cos \theta\left[1+e \cos \theta_{0}\right]}{2 \sqrt{\left(1+e \cos \theta_{0}\right)\left(1+e \cos \left(\theta-\theta_{0}\right)\right)}},
\end{aligned}
$$

where

$q(\theta)=8 \sin \theta+8 e(2+\cos \theta) \cos \frac{\theta-\theta_{0}}{2} \sin \frac{\theta}{2}+e^{2}\left(\cos \theta+3 \cos \left(\theta-2 \theta_{0}\right) \sin \theta\right)$. 
Now we compute the functions $\left(G_{1}, G_{2}\right)=M_{z}^{-1}(\theta) F_{1}(\theta, x(t ; z, 0))$ which appear in the next integral

$$
\left(g_{1}, g_{2}\right)=F(z)=\frac{1}{T} \int_{0}^{T} M_{z}^{-1}(t) F_{1}(t, x(t ; z, 0)) d t
$$

and we obtain

$$
\begin{aligned}
G_{1} & =\frac{p_{1}(\theta)}{8\left(1+e \cos \theta_{0}\right)^{3 / 2}\left(e^{2}-1\right)\left(1+e \cos \left(\theta-\theta_{0}\right)\right)} \\
G_{2} & =\frac{p_{2}(\theta)}{16\left(e^{2}-1\right)\left(1+e \cos \left(\theta-\theta_{0}\right)\right) \sqrt{1+e \cos \theta_{0}}}
\end{aligned}
$$

where

$$
\begin{aligned}
p_{1}(\theta)= & {\left[e^{2}-1-16 h+\left(e^{2}-1\right) \cos 2 \theta-16 e h \cos \left(\theta-\theta_{0}\right)\right] . } \\
& {\left[2 \cos \theta\left(1+e \cos \theta_{0}\right)+e \sin \theta \sin \theta_{0}\right] \cdot\left(1+e \cos \left(\theta-\theta_{0}\right)\right)-} \\
& \cos \theta \sin \theta\left(e^{2}-1\right)\left[16\left(1+e \cos \theta_{0}\right) \sin \theta+\right. \\
& e\left(\left(e+4 \cos \theta_{0}+3 e \cos 2 \theta_{0}\right) \sin 2 \theta+\right. \\
& \left.\left.16(2+\cos \theta) \sin ^{2} \frac{\theta}{2} \sin \theta_{0}\right)+6 e^{2} \sin ^{2} \theta \sin 2 \theta_{0}\right], \\
p_{2}(\theta)= & \sin \theta\left\{10\left(e^{2}-1\right)-16 h\left(e^{2}+2\right)+10\left(e^{2}-1\right) \cos 2 \theta+\right. \\
& e\left[2\left(5 e^{2}-32 h-5\right) \cos \left(\theta-\theta_{0}\right)-16 e h \cos \left(2 \theta-2 \theta_{0}\right)+\right. \\
& \left.\left(e^{2}-1\right)\left(3 \cos \left(3 \theta-\theta_{0}\right)+7 \cos \left(\theta+\theta_{0}\right)\right)\right] .
\end{aligned}
$$

Computing the integrals (12), we obtain

$$
\begin{aligned}
& g_{1}\left(e, \theta_{0}\right)=-\frac{p_{3}\left(e, \theta_{0}\right)}{16 \pi e^{3}\left(1+e \cos \theta_{0}\right)^{3 / 2}\left(1-e^{2}\right)^{3 / 2}}, \\
& g_{2}\left(e, \theta_{0}\right)=-\frac{p_{4}\left(e, \theta_{0}\right)}{8 e^{3}\left(1-e^{2}\right) \sqrt{1+e \cos \theta_{0}}},
\end{aligned}
$$

where

$$
\begin{aligned}
p_{3}= & -8 e^{4} \sqrt{1-e^{2}} h \pi\left[4 \cos \theta_{0}+e\left(\cos 2 \theta_{0}+3\right)\right]+ \\
& \pi\left(e^{2}-1\right)\left[e^{3}\left(\sqrt{1-e^{2}}+e^{2}-1\right)+4 e^{2}\left(\sqrt{1-e^{2}}+e^{2}-1\right) \cos \theta_{0}-\right. \\
& 6 e\left(2-2 \sqrt{1-e^{2}}+e^{2}\left(\sqrt{1-e^{2}}-2\right)\right) \cos 2 \theta_{0}-\left(4-4 \sqrt{1-e^{2}}+\right. \\
& \left.\left.e^{2}\left(e^{2}+3 \sqrt{1-e^{2}}-5\right)\right)\left(4 \cos 3 \theta_{0}+e \cos 4 \theta_{0}\right)\right], \\
p_{4}= & e^{2}\left(1-\sqrt{1-e^{2}}+e^{2}\left(\sqrt{1-e^{2}}-8 h-1\right)\right) \sin \theta_{0}+ \\
& \left(e^{2}-1\right)\left(4-4 \sqrt{1-e^{2}}+e^{2}\left(\sqrt{1-e^{2}}-3\right)\right) \sin 3 \theta_{0} .
\end{aligned}
$$


Let

$$
m\left(e, \theta_{0}\right)=\operatorname{det}\left(\begin{array}{ll}
\frac{\partial g_{1}\left(e, \theta_{0}\right)}{\partial e} & \frac{\partial g_{1}\left(e, \theta_{0}\right)}{\partial \theta_{0}} \\
\frac{\partial g_{2}\left(e, \theta_{0}\right)}{\partial e} & \frac{\partial g_{2}\left(e, \theta_{0}\right)}{\partial \theta_{0}}
\end{array}\right) .
$$

Now we have to study the solutions $\left(e, \theta_{0}\right)$ of system $g_{1}\left(e, \theta_{0}\right)=0, g_{2}\left(e, \theta_{0}\right)=$ 0 with $e \in(0,1)$. Note that if $e=0$ then $g_{1}\left(e, \theta_{0}\right) \equiv 0$ and $g_{2}\left(e, \theta_{0}\right) \equiv 0$, and that in this case the averaging theory does not provide information on the periodic solutions of system (8).

We first solve the equation $g_{2}\left(e, \theta_{0}\right)$ with respect to $\theta_{0}$, and we obtain ten solutions as follows:

$$
\begin{array}{ll}
\theta_{0}^{1}=0, & \theta_{0}^{2}=\pi, \\
\theta_{0}^{3}=-\arccos (-F(e)), & \theta_{0}^{4}=-\arccos (-F(e))+\pi, \\
\theta_{0}^{5}=\arccos (-F(e)), & \theta_{0}^{6}=\arccos (-F(e))+\pi, \\
\theta_{0}^{7}=-\arccos F(e), & \theta_{0}^{8}=-\arccos F(e)+\pi, \\
\theta_{0}^{9}=\arccos F(e), & \theta_{0}^{10}=\arccos F(e)+\pi,
\end{array}
$$

where

$$
F(e)=\frac{\sqrt{2\left[\left(1+\sqrt{1-e^{2}}\right)(1-16 h)-e^{2}\left(1+\sqrt{1-e^{2}}-4 h\left(3+\sqrt{1-e^{2}}\right)\right)\right]}}{2 e \sqrt{1-e^{2}}} .
$$

Of course, $g_{1}\left(\theta_{0}, e\right)=0$ if and only if $p_{3}\left(\theta_{0}, e\right)=0$.

Case 1: $\theta_{0}=\theta_{0}^{1}=0$. Substituting $\theta_{0}^{1}$ into $p_{3}\left(e, \theta_{0}\right)$, we obtain

$$
\bar{p}_{3}(e)=16(e-1)^{2}(e+1)^{3}-8(1+e) \sqrt{1-e^{2}}\left(2-3 e^{2}+e^{4}+4 e^{4} h\right) .
$$

We solve $\bar{p}_{3}(e)=0$ with respect to $h$, we have

$$
h(e)=-\frac{\left(1-e^{2}\right)\left(1-\sqrt{1-e^{2}}\right)^{2}}{4 e^{4}} .
$$

By simple computation, we have

$$
h^{\prime}(e)=\frac{4-4 \sqrt{1-e^{2}}+e^{2}\left(\sqrt{1-e^{2}}-3\right)}{2 e^{5}} .
$$

Let $f(e)=4-4 \sqrt{1-e^{2}}+e^{2}\left(\sqrt{1-e^{2}}-3\right)$, we have

$$
f^{\prime}(e)=\frac{3 e\left(1-\sqrt{1-e^{2}}\right)^{2}}{\sqrt{1-e^{2}}} .
$$

Note that $f(0)=0$, then by $(15)$, we deduce that $f(e)>0$ for all $0<e<1$. Then from (14), we have $h^{\prime}(e)>0$ for all $0<e<1$, so $h(e)$ is a strictly monotone function on $(0,1)$. Since $h(1)=0, h(e)<0$ in the interval $(0,1)$. 
Next we can compute $m\left(e, \theta_{0}^{1}\right)$ and we obtain

$$
-\frac{4 e^{2}\left(5-3 \sqrt{1-e^{2}}\right)+e^{4}\left(\sqrt{1-e^{2}}-5\right)+16\left(\sqrt{1-e^{2}}-1\right)}{2 e^{7}(1+e)\left(\sqrt{1-e^{2}}\right)} .
$$

Let $g(e)=4 e^{2}\left(5-3 \sqrt{1-e^{2}}\right)+e^{4}\left(\sqrt{1-e^{2}}-5\right)+16\left(\sqrt{1-e^{2}}-1\right)$, we have

$$
g^{\prime}(e)=-\frac{5 e\left(8-8 e^{2}+e^{4}-8 \sqrt{1-e^{2}}+4 e^{2} \sqrt{1-e^{2}}\right)}{\sqrt{1-e^{2}}} .
$$

Let $y(e)=8-8 e^{2}+e^{4}-8 \sqrt{1-e^{2}}+4 e^{2} \sqrt{1-e^{2}}$, we have

$$
y^{\prime}(e)=\frac{4 e(1-\sqrt{1-e})^{3}}{\sqrt{1-e^{2}}}>0,
$$

for $e \in(0,1)$. Note that $y(0)=0$, and from (17) and (18) we can deduce that $y(e)>0$ and $g^{\prime}(e)<0$ in the interval $(0,1)$. Then, since $g(0)=0$ we have $g(e)<0$ in the interval $(0,1)$. So, from (16), we have $m(e, 0)>0$ for $e \in(0,1)$. Therefore, from the averaging theory (see [5]) for every value of the eccentricity $e \in(0,1)$ and for $\varepsilon$ sufficiently small the periodic solution $(v(\theta ; e, 0), u(\theta ; e, 0))$ (given in $(10))$ of the Kepler problem such that $(v(0 ; e, 0), u(0 ; e, 0))=(0, \sqrt{1+e})$, can be continued to the energy level $h=h(e)<0$ of the anisotropic Manev problem (1).

Case 2: $\theta_{0}=\theta_{0}^{2}=\pi$. Working as in the previous case we obtain that the periodic solution $(v(\theta ; e, \pi), u(\theta ; e, \pi))$ which can be continued from the Kepler problem to the anisotropic Manev problem is the same that in Case 1 , because $(v(0 ; e, \pi), u(0 ; e, \pi))=(0, \sqrt{1+e})$, i.e. both periodic solutions have the same initial conditions.

Case 3: $\theta_{0}=\theta_{0}^{3}$. Now solving $\bar{p}_{3}(e)=p_{3}\left(e, \theta_{0}^{3}\right)=0$ with respect to $h$, we obtain the two solutions

$$
h_{ \pm}(e)=s_{ \pm}(e) \frac{1-e^{2}}{4 e^{6}},
$$

where $s_{ \pm}(e)$ is given by

$$
\begin{aligned}
& -e^{2}\left[4+e^{4}-4 \sqrt{1-e^{2}}+e^{2}\left(3 \sqrt{1-e^{2}}-5\right)\right] \pm \\
& \sqrt{-\left[e^{6}+e^{2}\left(48-32 \sqrt{1-e^{2}}\right)+6 e^{4}\left(\sqrt{1-e^{2}}-3\right)+32\left(\sqrt{1-e^{2}}-1\right)\right]} .
\end{aligned}
$$

Since substituting $\theta_{0}=\theta_{0}^{3}$ and $h=h_{ \pm}(e)$ into $m\left(e, \theta_{0}\right)$, we found $m\left(e, \theta_{0}^{3}\right)=0$ for all $e \in(0,1)$, the averaging theory does not provide information if the corresponding periodic solution of the Kepler problem $\left(v\left(\theta ; e, \theta_{0}^{3}\right), u\left(\theta ; e, \theta_{0}^{3}\right)\right)$ can be continued or not to the anisotropic Manev problem (1). 
For the cases $\theta_{0}^{j}$ for $j=4, \ldots, 10$, we also found that $m\left(e, \theta_{0}^{j}\right)=$ 0 for all $e \in(0,1)$, so again the averaging theory does not provide information about the possible continuation of this periodic solution to the anisotropic Manev problem (1).

In conclusion the averaging theory provides a unique periodic solution of the Kepler problem for each value of the eccentricity $e \in(0,1)$ which can be continued to the anisotropic Kepler problem (1). This completes the proof of Theorem 1 .

\section{Proof of Theorem 2}

In the McGehee coordinates the equations of motion (3) become

$$
\begin{aligned}
& r^{\prime}=r^{-1 / 2} v, \\
& \theta^{\prime}=r^{-3 / 2} u, \\
& v^{\prime}=r^{-3 / 2}\left(u^{2}+\frac{1}{2} v^{2}-1\right)+\varepsilon r^{\frac{1}{2}}\left(a \cos \theta+2 b r \cos ^{2} \theta\right), \\
& u^{\prime}=r^{-3 / 2}\left(-\frac{1}{2} u v\right)+\varepsilon r^{\frac{1}{2}}(a \sin \theta+2 b r \cos \theta \sin \theta),
\end{aligned}
$$

and the energy level $H=h$ writes

$$
\frac{1}{2}\left(u^{2}+v^{2}\right)-1+\varepsilon\left(a r^{2} \cos \theta+b r^{3} \cos ^{2} \theta\right)=r h .
$$

Then doing the change of time $t \rightarrow \tau$ given by $d t / d \tau=r^{3 / 2}$, the equations of motion (19) in the new time $\tau$ are

$$
\begin{aligned}
& \dot{r}=r v, \\
& \dot{\theta}=u, \\
& \dot{v}=u^{2}+\frac{1}{2} v^{2}-1+\varepsilon\left(a r^{2} \cos \theta+2 b r^{3} \cos ^{2} \theta\right), \\
& \dot{u}=-\frac{1}{2} u v-\varepsilon\left(a r^{2} \sin \theta+2 b r^{3} \cos \theta \sin \theta\right) .
\end{aligned}
$$

Note that these equations of motion are defined for all $(r, \theta, v, u) \in$ $[0,+\infty) \times \mathbb{S}^{1} \times \mathbb{R}^{2}$.

Computing $r$ from the energy level $H=h<0$, where $H$ is given in (20) we have

$$
r=r(\theta, v, u, h)=f_{0}+\varepsilon f_{1}+O\left(\varepsilon^{2}\right),
$$

where

$$
\begin{aligned}
& f_{0}=\frac{u^{2}+v^{2}-2}{2 h}, \\
& f_{1}=\frac{\left(u^{2}+v^{2}-2\right)^{2} \cos \theta\left[2 a h+b\left(u^{2}+v^{2}-2\right) \cos \theta\right]}{8 h^{4}} .
\end{aligned}
$$


Then the equations of motion (21) in the energy level $H=h<0$ become

$(22)$

$$
\begin{aligned}
& \frac{d v}{d \theta}=\frac{2 u^{2}+v^{2}-2}{2 u}-\varepsilon \frac{\left(u^{2}+v^{2}-2\right)^{2}\left[a h+b\left(u^{2}+v^{2}-2\right) \cos \theta\right] \cos \theta}{d u}+O\left(\varepsilon^{2}\right), \\
& \frac{d u}{d \theta}=-\frac{v}{2}+\varepsilon \frac{\left(u^{2}+v^{2}-2\right)^{2}\left[a h+b\left(u^{2}+v^{2}-2\right) \cos \theta\right] \sin \theta}{4 h^{3} u}+O\left(\varepsilon^{2}\right) .
\end{aligned}
$$

Since hydrogen atom problem (3) for $\varepsilon=0$ is the Kepler problem (9), as before we take $h$ negative because we want to continue the periodic solutions of the Kepler problem to periodic solutions of the hydrogen atom problem (3).

As in the previous problem we shall use the averaging theory for studying the periodic solutions of system (9) which can be continued to system (22).

We use the notation of [5] for applying the averaging theory, thus we have from system (22) that

$$
\begin{array}{ll}
F_{01}=\frac{2 u^{2}+v^{2}-2}{2 u}, & F_{11}=\frac{-16 h+\left(-4+2 u^{2}+2 v^{2}\right) \cos ^{2} \theta}{4 u\left(-2+u^{2}+v^{2}\right)}, \\
F_{02}=-\frac{v}{2}, & F_{12}=\frac{\cos \theta \sin \theta}{u} .
\end{array}
$$

Now we compute the functions $\left(G_{1}, G_{2}\right)=M_{z}^{-1}(\theta) F_{1}(\theta, x(t ; z, 0))$ which appear in the integral (12) and we obtain

$$
\begin{aligned}
G_{1} & =\frac{p_{1}(\theta)}{8 h^{3}\left(1+e \cos \left(\theta-\theta_{0}\right)\right)^{4}\left(1+e \cos \theta_{0}\right)^{\frac{3}{2}}}, \\
G_{2} & =\frac{p_{2}(\theta)}{8 h^{3}\left(1+e \cos \left(\theta-\theta_{0}\right)\right)^{4} \sqrt{1+e \cos \theta_{0}}}
\end{aligned}
$$

where

$$
\begin{aligned}
p_{1}(\theta)= & \left(e^{2}-1\right)^{2}\left(b\left(e^{2}-1\right) \cos \theta+a h\left(1+e \cos \left(\theta-\theta_{0}\right)\right) .\right. \\
& \left(\cos 2 \theta-3-2 e \cos \theta_{0}\left(2+\cos \theta\left(1-\cos \theta+e \cos \theta_{0}\right)\right)+\right. \\
& \left.e\left(\cos \theta-4-3 e \cos \theta_{0}\right) \sin \theta \sin \theta_{0}\right), \\
p_{2}(\theta)= & \left(e^{2}-1\right)^{2}\left(b\left(e^{2}-1\right) \cos \theta+a h\left(1+e \cos \left(\theta-\theta_{0}\right)\right)\left(\cos \theta+e \cos \theta_{0}\right) \sin \theta,\right.
\end{aligned}
$$


Computing the integrals (12) for the hydrogen atom problem (22) we get

$$
\begin{aligned}
& g_{1}\left(e, \theta_{0}\right)=\frac{\sqrt{1-e^{2}}\left(7 b e^{2}+12 a h+4 e(4 b+3 a h) \cos \theta_{0}+9 b e^{2} \cos 2 \theta_{0}\right)}{32 h^{3}\left(1+e \cos \theta_{0}\right)^{\frac{3}{2}}}, \\
& g_{2}\left(e, \theta_{0}\right)=\frac{b e \sin \theta_{0} \sqrt{1-e^{2}}}{16 h^{3} \sqrt{1+e \cos \theta_{0}}} .
\end{aligned}
$$

Now we have to study the solutions $\left(e, \theta_{0}\right)$ of the system $g_{1}\left(e, \theta_{0}\right)=$ $0, g_{2}\left(e, \theta_{0}\right)=0$ with $e \in(0,1)$, as before $e$ cannot be zero.

First we solve the equation $g_{2}\left(e, \theta_{0}\right)$ with respect to $\theta_{0}$, and we obtain two solutions

$$
\theta_{0}^{1}=0, \quad \theta_{0}^{2}=\pi .
$$

Case 1: $\theta_{0}=\theta_{0}^{1}=0$. Substituting $\theta_{0}^{1}$ into $g_{1}\left(e, \theta_{0}\right)$, we have

$$
\bar{g}_{1}(e)=\frac{\sqrt{1-e^{2}}\left[16 b e^{2}+12 a h+4 e(4 b+3 a h)\right]}{32 h^{3}(1+e)^{\frac{3}{2}}} .
$$

We solve $\bar{g}_{1}(e)=0$ with respect to $e$, we have

$$
e=1, \quad e=-\frac{3 a h}{4 b}
$$

The solution $e=1$ should be eliminated since we study which periodic solutions of the Kepler problem can be continued to periodic solutions of the perturbed hydrogen atom problem, and fore $=1$ the solutions of the Kepler problem are parabolas.

By direct computation we have $m\left(-\frac{3 a h}{4 b}, 0\right)=-\frac{3 a b}{512 h^{5}}\left|\frac{4 b+3 a h}{b}\right|$. Therefore, from the averaging theory we obtain that if the parameters $a$ and $b$ of the perturbation satisfy $0<-3 a h / 4 b<1, m(-3 a h / 4 b, 0) \neq 0$ and $\varepsilon \neq 0$ is sufficiently small, then the periodic solution

$$
\left(v\left(\theta ;-3 a b /\left(512 h^{5}\right), 0\right), u\left(\theta ;-3 a b /\left(512 h^{5}\right), 0\right)\right)
$$

of the Kepler problem can be continued to the energy level $h<0$ of the perturbed hydrogen atom problem (3).

Case 2: $\theta_{0}=\theta_{0}^{2}=\pi$. Substituting $\theta_{0}^{2}$ into $g_{1}\left(e, \theta_{0}\right)$, we obtain

$$
\bar{g}_{1}(e)=\frac{\sqrt{1-e^{2}}\left[16 b e^{2}+12 a h-4 e(4 b+3 a h)\right]}{32 h^{3}(1-e)^{\frac{3}{2}}} .
$$

We solve $\bar{g}_{1}(e)=0$ with respect to $e$ and we get

$$
e=-1, \quad e=\frac{3 a h}{4 b}
$$


Since $m\left(\frac{3 a h}{4 b}, \pi\right)=\frac{3 a b}{512 h^{5}}\left|\frac{4 b+3 a h}{b}\right|$, as before from the averaging theory we obtain that if the parameters $a$ and $b$ of the perturbation satisfy $3 a h / 4 b \in(0,1), m(3 a h /(4 b), \pi) \neq 0$ and for $\varepsilon \neq 0$ is sufficiently small, then the periodic solution of the Kepler problem

$$
(v(\theta ; 3 a b /(4 b), \pi), u(\theta ; 3 a b /(4 b), \pi))
$$

can be continued to the energy level $h<0$ of the perturbed hydrogen atom problem (3). This completes the proof of Theorem 2.

\section{Proof of Theorem 3}

The proof of Theorem 3 is similar to the proof of Theorem 2 .

We also take McGehee coordinates for the equations of motion (4) and doing the change of time $t \rightarrow \tau$ given by $d t / d \tau=r^{3 / 2}$, the equations of motion (4) in the new time $\tau$ become

$$
\begin{aligned}
& \dot{r}=r v, \\
& \dot{\theta}=u, \\
& \dot{v}=u^{2}+\frac{1}{2} v^{2}-1+\varepsilon\left(a r^{2} \cos \theta+2 b r^{3} \sin ^{2} \theta\right), \\
& \dot{u}=-\frac{1}{2} u v-\varepsilon\left(a r^{2} \sin \theta-2 b r^{3} \cos \theta \sin \theta\right) .
\end{aligned}
$$

and the energy level $H=h$ becomes

$$
\frac{1}{2}\left(u^{2}+v^{2}\right)-1+\varepsilon\left(a r^{2} \cos \theta+b r^{3} \sin ^{2} \theta\right)=r h .
$$

From this energy relation $H=h<0$ we can compute

$$
r=r(\theta, v, u, h)=f_{0}+\varepsilon f_{1}+O\left(\varepsilon^{2}\right),
$$

where

$$
\begin{aligned}
& f_{0}=\frac{u^{2}+v^{2}-2}{2 h}, \\
& f_{1}=\frac{\left(u^{2}+v^{2}-2\right)^{2}\left[2 a h \cos \theta+b\left(u^{2}+v^{2}-2\right) \sin ^{2} \theta\right]}{8 h^{4}} .
\end{aligned}
$$

Then the equations of motion (23) restricted to the energy level $H=$ $h<0$ are

$$
\begin{aligned}
& \frac{d v}{d \theta}=\frac{2 u^{2}+v^{2}-2}{2 u}-\varepsilon \frac{\left(u^{2}+v^{2}-2\right)^{2}\left[a h \cos \theta+b\left(u^{2}+v^{2}-2\right) \sin ^{2} \theta\right]}{4 h^{3} u}+O\left(\varepsilon^{2}\right), \\
& \frac{d u}{d \theta}=-\frac{v}{2}+\varepsilon \frac{\left(u^{2}+v^{2}-2\right)^{2}\left[a h-b\left(u^{2}+v^{2}-2\right) \cos \theta\right] \sin \theta}{4 h^{3} u}+O\left(\varepsilon^{2}\right) .
\end{aligned}
$$


The unperturbed system when $\varepsilon=0$ is again the Kepler problem given in (9) having the general solution provided in (10).

Using the notation of [5] we have for system (23) that

$$
\begin{aligned}
& F_{01}=\frac{2 u^{2}+v^{2}-2}{2 u}, \quad F_{11}=-\frac{v}{2}, \\
& F_{02}=-\frac{\left(u^{2}+v^{2}-2\right)^{2}\left[a h \cos \theta+b\left(u^{2}+v^{2}-2\right) \sin ^{2} \theta\right]}{4 h^{3} u}, \\
& F_{12}=\frac{\left(u^{2}+v^{2}-2\right)^{2}\left[a h-b\left(u^{2}+v^{2}-2\right) \cos \theta\right] \sin \theta}{4 h^{3} u} .
\end{aligned}
$$

Now we compute the functions $\left(G_{1}, G_{2}\right)=M_{z}^{-1}(\theta) F_{1}(\theta, x(t ; z, 0))$ which appear in the integral (12) and we obtain

$$
\begin{aligned}
G_{1} & =\frac{p_{1}(\theta)}{32 h^{3}\left(1+e \cos \left(\theta-\theta_{0}\right)\right)^{5}\left(1+e \cos \theta_{0}\right)^{\frac{3}{2}}}, \\
G_{2} & =\frac{p_{2}(\theta)}{16 h^{3}\left(1+e \cos \left(\theta-\theta_{0}\right)\right)^{4} \sqrt{1+e \cos \theta_{0}}},
\end{aligned}
$$

where

$$
\begin{aligned}
p_{1}(\theta)= & \left(1-e^{2}\right)^{2}\left(-4\left(a h \cos \theta\left(1+e \cos \left(\theta-\theta_{0}\right)\right)^{2}+b\left(e^{2}-1\right) \sin ^{2} \theta\right) .\right. \\
& \left(2 \cos \theta\left(1+e \cos \theta_{0}\right)+e \sin \theta \sin \theta_{0}\right)-\left(\left(b\left(1-e^{2}\right) \cos \theta+\right.\right. \\
& \left.a h\left(1+e \cos \left(\theta-\theta_{0}\right)\right)\right) \sin \theta\left(16\left(1+e \cos \theta_{0}\right) \sin \theta+\right. \\
& e\left(\left(e+4 \cos \theta_{0}+3 e \cos 2 \theta_{0}\right) \sin 2 \theta+16(2+\cos \theta) \sin ^{2} \frac{\theta}{2} \sin \theta_{0}\right)+ \\
& \left.\left.\left.6 e^{2} \sin ^{2} \theta \sin 2 \theta_{0}\right)\right)\right), \\
p_{2}(\theta)= & \left(e^{2}-1\right)^{2}\left(3 b-3 b e^{2}+a\left(e^{2}+2\right) h \cos \theta-b\left(e^{2}-1\right) \cos 2 \theta+\right. \\
& a e^{2} h \cos \left(\theta-2 \theta_{0}\right)+3 b e \cos \left(\theta-\theta_{0}\right)-3 b e^{3} \cos \left(\theta-\theta_{0}\right)+ \\
& a e h \cos \left(2 \theta-\theta_{0}\right)+3 a e h \cos \theta_{0}+b e \cos \left(\theta+\theta_{0}\right)- \\
& \left.b e^{3} \cos \left(\theta+\theta_{0}\right)\right) \sin \theta,
\end{aligned}
$$

Computing the integrals (12) for our system (23) we get

$$
\begin{aligned}
& g_{1}=\frac{\sqrt{1-e^{2}}\left[2 e(b-3 a h) \cos \theta_{0}+3 b e^{2} \cos 2 \theta_{0}-b e^{2}-6 a h\right]}{16 h^{3}\left(1+e \cos \theta_{0}\right)^{3 / 2}}, \\
& g_{2}=-\frac{b e \sin \theta_{0} \sqrt{1-e^{2}}}{4 h^{3} \sqrt{1+e \cos \theta_{0}}} .
\end{aligned}
$$

Now we have to study the solutions $\left(e, \theta_{0}\right)$ of the system $g_{1}\left(e, \theta_{0}\right)=$ $0, g_{2}\left(e, \theta_{0}\right)=0$ with $e \in(0,1)$. We first solve the equation $g_{2}\left(e, \theta_{0}\right)$ 
with respect to $\theta_{0}$, and we obtain the two solutions

$$
\theta_{0}^{1}=0, \quad \theta_{0}^{2}=\pi
$$

Case 1: $\theta_{0}=\theta_{0}^{1}=0$. Substituting $\theta_{0}^{1}$ into $g_{1}\left(e, \theta_{0}\right)$, we get

$$
\bar{g}_{1}(e)=\frac{\sqrt{1-e^{2}}\left(2 b e^{2}+2 e(b-3 a h)-6 a h\right)}{16 h^{3}(1+e)^{\frac{3}{2}}} .
$$

We solve $\bar{g}_{1}(e)=0$ with respect to $e$, we have

$$
e=1, \quad e=\frac{3 a h}{b} .
$$

By direct computations we have $m\left(\frac{3 a h}{b}, 0\right)=-\frac{3 a b}{32 h^{5}}\left|\frac{b-3 a h}{b}\right|$. Therefore for every $e=3 a h / b \in(0,1), m(3 a h / b, 0) \neq 0$ and for $\varepsilon \neq 0$ sufficiently small the periodic solution of the Kepler problem

$$
(v(\theta ; 3 a b / b, 0), u(\theta ; 3 a b / b, 0))
$$

can be continued to the energy level $h<0$ of the perturbed hydrogen atom problem (4).

Case 2: $\theta_{0}=\theta_{0}^{2}=\pi$. Substituting $\theta_{0}^{2}$ into $g_{1}\left(e, \theta_{0}\right)$, we obtain

$$
\bar{g}_{1}(e)=\frac{\sqrt{1-e^{2}}\left(2 b e^{2}-2 e(b-3 a h)-6 a h\right)}{16 h^{3}(1-e)^{\frac{3}{2}}} .
$$

We solve $\bar{g}_{1}(e)=0$ with respect to $e$, we have

$$
e=-1, \quad e=-\frac{3 a h}{b} \text {. }
$$

Since $m\left(-\frac{3 a h}{b}, \pi\right)=\frac{3 a b}{32 h^{5}}\left|\frac{b-3 a h}{b}\right|$, for every value of the eccentricity $e=-3 a h / b \in(0,1), m(-3 a h / b, \pi) \neq 0$ and for $\varepsilon \neq 0$ sufficiently small the periodic solution of the Kepler problem

$$
(v(\theta ; 3 a b / b, \pi), u(\theta ; 3 a b / b, \pi))
$$

can be continued to the energy level $h<0$ of the perturbed hydrogen atom problem (4). This completes the proof of Theorem 3.

\section{ACKNOWLEDGEMENTS}

The first author is partially supported by the Ministerio de Economía, Industria y Competitividad, Agencia Estatal de Investigación grants MTM2016-77278-P (FEDER) and MDM-2014-0445, the Agència de 
Gestió d'Ajuts Universitaris i de Recerca grant 2017SGR1617, and the H2020 European Research Council grant MSCA-RISE-2017-777911.

The second author is partially supported by Fundamental Research Funds for the Central Universities (NO.XDJK2015C139), China Scholarship Council(N0.201708505030).

\section{REFERENCES}

[1] Abouelmagd, E.I., Llibre, J., Garcia Guirao, J.L., Periodic orbits of the planar anisotropic kepler probelm, Internat. J. Bifur. Chaos Appl. Sci. Engrg., 27 (2017), 1750039-1, pp 1-6.

[2] Aparicio, I. and Floría, L., Canonical focal method treatment of a GyldenManeff problem, Posters of the IV Catalan Days of Applied Mathematics, (1998), 1-16.

[3] Barrabés E., Ollé M., Borondo F., Farrelly D., Mondelo J.M., Phase space structure of the hydrogen atom in a circularly polarized microwave field, Phys. D, 241(4) (2012), 333-349.

[4] Brunello A.F., Uzer T., Farrelly D., Hydrogen atom in circularly polarized microwaves, chaotic ionization via core scattering, Phys. Rev. 55(5) (1997), 3730-3745.

[5] Buica, A., Francoise, J.P., Llibre, J., Periodic solutions of nonlinear periodic differential systems with a small parameter, Commun.Pure Appl. Anal., 6 (2007), 103-111.

[6] Craig, S., Diacu, F.N., Lacomba, E.A. and Perez, E., On the anisotropic Manev problem, J. Math. Phys., 40 (1999), 1359-1375.

[7] Cushman RH., van der Meer J.C., Orbiting dust under radiation pressure, In: Berger HB, Hennig JD, editors. Proceedings of the Fifteenth International Conference on Differential Geometric Methods in Theoretical Physics, World Scientific, Germany, 1986, pp 403-414.

[8] Delgado, J., Diacu, F.N., Lacomba, E.A., Mingarelli, A., Mioc, V., Perez, E., Stoica, C., The global flow of the Manev problem, J. Math. Phys., 37 (1996), 2748-2761.

[9] Deprit A., Dynamics of orbiting dust under radiation pressure. In: Berger A, editor. The Big Bang and Georges Lemaitre. Dordrecht: D. Reidel, 1984, pp 151-180.

[10] Devaney, R.L., Blowing up singularities in classical mechanical systems, Amer. Math. Monthly, 89 (1982), no. 8, 535-552.

[11] Devaney, R. L., Singularities in classical mechanical systems, Ergodic theory and dynamical systems, I (College Park, Md., 1979 C80), 211-333, Progr. Math., vol. 10, Birkhauser, Boston, Mass., 1981.

[12] Diacu, F.N., The planar isosceles problem fro Maneff's gravitational law, J. Math. Phys., 34 (1993), 5671-5690.

[13] Farrelly D., Uzer T., Ionization mechanism of Rydberg atoms in a circularly polarized microwave field, Phys. Rev. Lett., 74(10) (1995), 1720-1723.

[14] Fu P., Scholz T.J., Hettema J.M., Gallagher T.F., Ionization of Rydberg atoms by a circularly polarized microwave field, Phys. Rev. Lett., 64(5) (1995), 511514 . 
[15] Gebarowski R., Zakrzewski J., Ionization of hydrogen atoms by circularly polarized microwaves, Phys. Rev. A, 51 (1995), 1508-1519.

[16] Griffiths J.A., Farrelly D., Ionization of Rydberg atoms by circularly and elliptically polarized microwave fields, Phys. Rev. A, 45(5) (1992), 2678-2681.

[17] Lacomba, E.A., Llibre, J., Nunes, A., Invariant tori and cylinders for a class of perturbed Hamiltonian systems, in The Geometry of Hamiltonian Systems (Berkeley, CA, 1989) (Math. Sci. Res. Inst. Publ. vol 22), New York: Springer, 373-385.

[18] Lanchares V., Iãrrea M., Salas J.P., Bifurcations in the hydrogen atom in the presence of a circularly polarized microwave field and a static magnetic field, Phys. Rev. A, 56(3) (1997), 1839-1843.

[19] Nauenberg M., Comment on "Ionization of Rydberg atoms by a circularly polarized microwave field", Phys. Rev. Lett., 64 (1990), 27-31.

[20] Maneff, G., Die gravitation und das prinzip von wirkung und gegenwirkung, Z. Phys., 31 (1925), 786-802.

[21] Maneff, G., La gravitation et le principe de l'égalité de l'action et de la réaction, Comptes Rendus, 178, (1924), 2159-2161.

[22] Maneff, G., La gravitation et l'énergie au zéro, Comptes Rendus, 190 (1930), 1374-1377.

[23] Maneff, G., Le principe de la moindre action et la gravitation, Comptes Rendus, 190 (1930), 963-965.

[24] McGehee, R., Singularities in classical celestial mechanics, Proc. Int. Congr. Mathematicians 1978, Helsinki, 1980, pp. 827-834.

[25] Mioc, V., Elliptic-type motion in Fock's gravitational field, Astron. Nachr., 315 (1994), 175-180.

[26] Mioc, V., Radu, E., Orbits in an anisotropic radiation field, Astron. Nachr., 313 (1992), 353-357.

[27] Mioc, V., Stoica, C., Discussion et résolution compléte du probléme des deux corps dans le champ gravitationnel de Maneff, C. R. Acad. Sci., 320 (1995), 645-648.

[28] Mioc, V., Stoica, C., Discussion et résolution compléte du probl'eme des deux corps dans le champ gravitationnel de Maneff, II C. R. Acad. Sci., 321 (1995), 961-964.

[29] Moulton, F.R., An Introduction to Celestial Mechanics, 2nd Revised Edn, New York, Dover, 1970.

[30] Ollé M., To and fro motion for the hydrogen atom in a circularly polarized microwave field, Commun Nonlinear Sci Numer Simul, 54 (2017), 286-301.

[31] Ollé M., Pacha J.R., Hopf bifurcation for the hydrogen atom in a circularly polarized microwave field, Commun. Nonlinear Sci. Numer. Simul., 62 (2018), 27-60.

[32] Rakovic M.J., Chu S.I., Approximate dynamical symmetry of hydrogen atomsin circularly polarized microwave fields, Phys. Rev. A 50(6) (1994), 50775080 .

[33] Rakovic M.J., Chu S.I., New integrable systems: hydrogen atom in external fields, Phys. D, 81(3) (1995), 207-316.

[34] Roy A.E., Orbit Motion, CRC Press, 4th Edition, 2004.

[35] Saslaw W.C., Motions around a source whose luminosity changes, Astrophys. J., 226 (1978), 240-252. 
[36] Selaru D., Cucu-Dumitrescu C., Mioc V., On a two-body problem with periodically changing equivalent gravitational parameter, Astron. Nachr., 313 (1992), 257-263.

[37] Stoica C., Mioc V., On the two-body problem in Maneff-type fields, Bull. Astron. Belgrade, 154 (1996), 1-8.

[38] Ureche V., Free-fall collapse of a homogeneous sphere in Maneff's gravitational field, Rom. Astron. J., 5 (1995),145-148.

[39] Zakrzewski J., Delande D., Gay J.C., Ionization of hoghly excited hydrogen atoms by a circularly polarized microwave field, Phys. Rev. A, 47(4) (1993), $2468-2471$.

${ }^{1}$ Departament de Matemàtiques, Universitat Autònoma de Barcelona, 08193 Bellaterra, Barcelona, Catalonia, Spain

E-mail address: jllibre@mat.uab.cat

2 School of Mathematics and Statistics, Southwest University, 400715, Chongqing, China

E-mail address: yuanpengfei@swu.edu.cn 